\title{
Preliminary results of anatomic lung resection using energy-based tissue and vessel coagulative fusion technology
}

\author{
Matthew J. Schuchert, MD, Ghulam Abbas, MD, Brian L. Pettiford, MD, James D. Luketich, MD, and \\ Rodney J. Landreneau, MD
}

\begin{abstract}
Objectives: Mechanical stapling devices have been established as the mainstay of therapy in the selective isolation and division of bronchial and vascular structures during anatomic lung resection. Few data are available regarding the application of energy-based tissue fusion technology during anatomic lung resection. In the present study, we evaluated the use of energy-based instruments for the division of the pulmonary arterial and venous branches during anatomic lung resection.
\end{abstract}

\begin{abstract}
Methods: Anatomic lung resection (segmentectomy or lobectomy) was performed using energy-based coagulative fusion technology. A low-profile jaw can be used to facilitate dissection in both open and video-assisted thoracic surgery cases, applying a seal $6 \mathrm{~mm}$ wide by $22 \mathrm{~mm}$ in length. Two energy applications were applied to the arterial and venous branches before vessel division.

Results: The bipolar tissue fusion system was used in 211 patients between 2008 and 2010 (104 lobectomies and 107 anatomic segmentectomies). Initially, we used a device with a smaller, curved jaw $(\mathrm{n}=12)$, producing a 3.3to $4.7-\mathrm{cm}$ seal. No arterial dehiscences and 2 partial venous dehiscences that were recognized and controlled intraoperatively occurred. For the remaining cases, we used a new device with a larger jaw that applied a seal $6 \mathrm{~mm}$ wide by $22 \mathrm{~mm}$ in length. No arterial or venous dehiscences (vessel size range, 0.4-1.2 cm) occurred.

Conclusions: The bipolar tissue fusion system provided safe and reliable control of pulmonary arterial and venous branches during anatomic lung resection. The use of energy-based tissue fusion technology represents a reasonable alternative to mechanical stapling devices during anatomic lung resection. (J Thorac Cardiovasc Surg 2010;140:1168-73)
\end{abstract}

The standard approach to ligation and division of the pulmonary vasculature during anatomic lung resection includes mechanical stapler application using endosurgical and open stapling devices ${ }^{1}$ or manual suture ligation and division. $^{2}$ Although both approaches have been proved to be reliable and effective, ${ }^{3}$ the manual suture ligation technique can be associated with an increased operative time and the potential for ligature failure. ${ }^{4,5}$ The mechanical stapling approach can also be associated with stapler failure, resulting in life-threatening bleeding, ${ }^{5}$ pulmonary parenchymal contusion, or staple line dehiscence, leading to hematoma or bronchopleural fistula. ${ }^{6}$ In addition, the

From the Division of Thoracic and Foregut Surgery, Heart, Lung, and Esophageal Surgery Institute, University of Pittsburgh Medical Center Health System, Pittsburgh, $\mathrm{Pa}$.

Supported by the Heart, Lung, and Esophageal Surgery Institute, University of Pittsburgh Medical Center Health System, Pittsburgh, Pa.

Disclosures: None. The authors have no commercial ties to Valley Labs, the manufacturer of the device described in this report.

Read at the 90th Annual Meeting of The American Association for Thoracic Surgery, Toronto, Ontario, Canada, May 1-5, 2010.

Received for publication May 9, 2010; revisions received June 2, 2010; accepted for publication June 20, 2010; available ahead of print Sept 20, 2010.

Address for reprints: Matthew J. Schuchert, MD, Heart, Lung and Esophageal Surgery Institute, University of Pittsburgh Medical Center Health System, Shadyside Medical Building, Suite 715, 5200 Centre Ave, Pittsburgh, PA 15232 (E-mail: schuchertmj@upmc.edu).

0022-5223/\$36.00

Copyright (c) 2010 by The American Association for Thoracic Surgery doi:10.1016/j.jtcvs.2010.06.064 mechanical stapling devices require the use of multiple cartridge firings to accomplish pulmonary resection and can be associated with increased operative costs. ${ }^{7}$

Energy-based coagulative and tissue fusion technology has been applied effectively in the ligation and division of vessels and tissues in a wide variety of intra-abdominal procedures. ${ }^{8}$ To date, limited data are available regarding the application of energy-based techniques in the division of the pulmonary vasculature. Therefore, the goal of the present investigation was to evaluate the safety and efficacy of an electrical thermocoagulative device in accomplishing segmental pulmonary vasculature fusion and division during anatomic pulmonary resection. The outcome variables included success of vessel fusion, operative data, length of stay, morbidity, and mortality.

\section{PATIENTS AND METHODS Patients}

The institutional review board of the University of Pittsburgh approved the present study (PRO 10010260). Between 2008 and 2010, 211 consecutive anatomic lung resections using an energy-based tissue fusion device for division of the pulmonary vasculature were performed and served as the patient set for the present analysis. Anatomic segmentectomy was performed in 107 patients (79 video-assisted thoracic surgery [VATS] and 28 open procedures), with the remaining 104 patients undergoing lobectomy (51 VATS and 53 open). The patient demographics and tumor characteristics are listed in Table 1. 


\section{Abbreviation and Acronym}

VATS $=$ video-assisted thoracic surgery

\section{Operative Technique}

Anatomic segmentectomy $(n=107)$ and lobectomy $(n=104)$ were performed, as described previously. ${ }^{9}$ A VATS approach was used in 79 segments and (74\%) 51 lobes (49\%), with the VATS approach constituting $62 \%$ of all resections. The primary determinants in selecting a patient for segmentectomy were limited functional status, previous pulmonary resection, underlying patient comorbidities, and surgeon judgment regarding resectability using a segmental approach (small, peripheral lesions confined to a discrete bronchopulmonary segment). Lobar resections were performed in a standard fashion. Anatomic segmentectomies were performed as follows: right upper lobe (apical, anterior, posterior, or apicoposterior segments), right middle lobe (medial or lateral segments), right lower lobe (superior or basilar segments), left upper lobe (upper division or lingular segments), and right lower lobe (superior or basilar segments).

\section{Vascular Fusion and Division}

Electrical thermocoagulative fusion of the pulmonary vessels was performed using the LigaSure device (Valley Lab, Boulder, Colo) (Figure 1). It is currently the only Food and Drug Administration-approved energy device for division of the thoracic vasculature. This device is fit with a central cutting blade between the 2 coagulative surfaces of the instrument, promoting effective coagulative ligation (or sealing) and division of the pulmonary vessel or parenchyma. The system creates a vessel ligation (seal) by application of bipolar electrosurgical radiofrequency energy (coagulation) to vessels interposed between the jaws of the device. When applied to a vessel or tissue bundle, the radiofrequency energy reorganizes the collagen and elastin structure within the tissues using a combination of heat and pressure to achieve tissue fusion, thereby forming a permanent seal. An advantage of this system is the creation of sufficient, consistent jaw closure force for reliable, consistent tissue fusion. The microprocessor in the generator monitors the tissue properties, stops the application of energy, and allows a brief period of cooling before indicating that the seal cycle has been completed.

Two hand-held instruments were used during the present series (Figure 1). The LigaSure Impact $(\mathrm{n}=12)$ is $18 \mathrm{~cm}$ in length with a $14^{\circ}$ curved jaw. The seal width measures 3.3 to $4.7 \mathrm{~mm}$. The LigaSure Atlas $(\mathrm{n}=199)$ instrument measures $20 \mathrm{~cm}$ in length, facilitating use in both open and VATS cases. The low-profile jaw can be used to facilitate dissection and applies a seal $6 \mathrm{~mm}$ wide by $22 \mathrm{~mm}$ in length. An insulated layer minimizes heat transmission to the exterior jaw surfaces, with the thermal spread typically limited to $2 \mathrm{~mm}$. This device has been approved by the Food and Drug Administration for fusion and division of lymphatics, tissue bundles, and pulmonary vasculature up to $7 \mathrm{~mm}$ in size.

Two energy applications are typically applied before vessel division. Both pulmonary arterial and venous branches were divided using this technique. For pulmonary veins, we found division to be safer by taking the first-order branches of the vein, instead of the main trunk. Similarly, for arteries, we have generally divided primary pulmonary arteries up to $7 \mathrm{~mm}$ in diameter. For larger vessels, vascular division was achieved at the level of the first-order branches. We currently do not advocate the use of the LigaSure device for primary parenchymal division during segmentectomy and, instead, perform a stapled, extended segmentectomy encompassing the intersegmental planes with $3.5-\mathrm{mm}$ and 4.5 -mm staplers. ${ }^{9}$

\section{Hospital Course}

All patients were monitored carefully in the intensive care unit overnight and were transferred to the floor the next day. Strict attention was paid to chest tube outputs, perioperative hemoglobin trends, and serial chest radio-
TABLE 1. Patient and tumor characteristics

\begin{tabular}{lc}
\hline & Patients $(\mathbf{n}=\mathbf{2 1 1})$ \\
\hline Age & \\
Mean & 64.8 \\
Range & $19-88$ \\
Gender & $116 \mathrm{~F}, 95 \mathrm{M}$ \\
Histology & \\
Adenocarcinoma & $79(37 \%)$ \\
Squamous cell CA & $42(20 \%)$ \\
Large cell CA & $9(4 \%)$ \\
Other lung CA & $27(13 \%)$ \\
Metastatic cancer & $24(12 \%)$ \\
Benign & $30(14 \%)$ \\
Tumor Size & \\
Mean (cm) & 2.9 \\
Range $(\mathrm{cm})$ & $0.5-14.3$ \\
Approach & \\
VATS & $130(62 \%)$ \\
Open & $81(38 \%)$ \\
\hline
\end{tabular}

$F$, female; $M$, male.

graph findings. Chest tube management and discharge planning were individualized according to the patient clinical characteristics and surgeon judgment. Chest tubes were removed when air leaks had ceased and drainage had decreased to less than $250 \mathrm{~mL} /$ day. The patients were discharged after chest tube removal and when clinically stable. The discharge criteria were not influenced by the clinical pathways or the type of surgery performed.

\section{Study End Points}

The primary end point for the present analysis was the success of vascular division using a bipolar tissue fusion device. The secondary end points included the operative time, estimated blood loss, chest tube duration, length of stay, morbidity, and mortality.

\section{Follow-up}

Perioperative data were collected from the hospital chart, anesthesia, and operating room records, as well as from the electronic medical record for each patient. All patients were followed up postoperatively at 2 weeks, at 4- to 6-month intervals for the first 2 years, and yearly thereafter with computed tomography scans. The mean follow-up was 11.5 months.

\section{RESULTS}

\section{Patient and Tumor Characteristics}

The patient and tumor characteristics are summarized in Table 1. The mean patient age was 64.8 years (range, 19-88 years). Of the 211 patients, 95 were men and 116 were women. The final pathologic findings demonstrated cancer in $74 \%$, metastases in $12 \%$, and benign disease in $14 \%$. The mean lesion size was $2.9 \mathrm{~cm}$. Adenocarcinoma constituted the most common pathologic subtype of non-small cell lung cancer (37\% of cases), followed by squamous cell carcinoma (20\% of cases). All lobar and segmental resections were performed successfully using energybased vascular division.

\section{Perioperative Outcomes}

Initially, we used a device with a smaller, curved jaw (LigaSure Impact; $n=12$ cases), which produced a seal of 3.3 


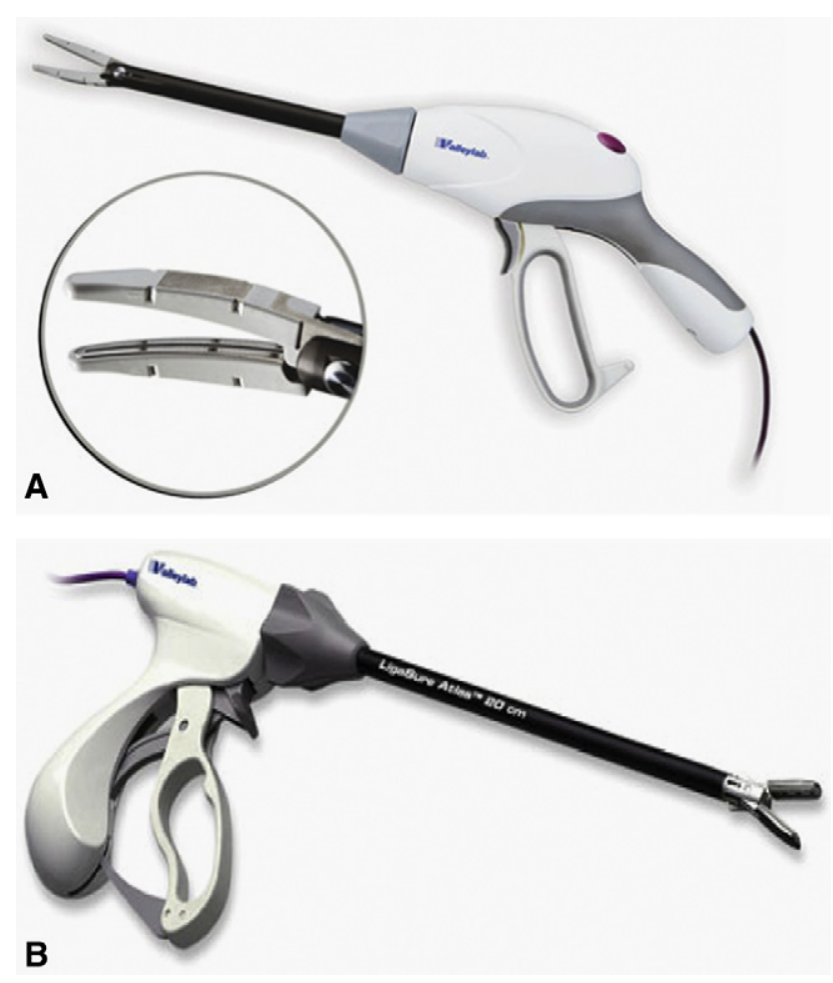

FIGURE 1. LigaSure device handpieces. A, LigaSure Impact. B, LigaSure Atlas.

to $4.7 \mathrm{~cm}$. No arterial dehiscences occurred, and 2 partial venous dehiscences developed that were recognized and controlled intraoperatively. The pulmonary veins have comparatively thinner vessel walls and associated reduced collagen content. The graded diameter of the curved tip did not seemingly achieve a seal as durable as that achieved with the thicker pulmonary arterial vessels. Therefore, for the remaining cases, we used a new device with a larger, higher pressure jaw that applies a seal $6 \mathrm{~mm}$ wide by $22 \mathrm{~mm}$ in length (LigaSure Atlas). No arterial or venous dehiscences occurred in vessels ranging from 0.4 to $1.2 \mathrm{~cm}$. The wider seal width $(6 \mathrm{~mm})$ appeared to enhance the vascular fusion integrity. Vascular division was successfully performed on 476 arterial and 229 venous branches without the need for hemostatic adjuncts (eg, clips or staples) The mean number of arteries divided was 2.3 per case and the mean number of veins was 1.1 per case. No cases of delayed hemorrhage occurred. The median operative time was 60 minutes. The median estimated blood loss was $100 \mathrm{~mL}$ (range, $10-800 \mathrm{~mL}$ ). The median chest tube duration was 5 days, and the length of stay was 6 days (Table 2). In addition to a prolonged air leak (defined as a persistent air leak for $>5$ days; $n=12,5.7 \%$ ), the observed chest tube duration was influenced by several variables, including transient air leaks ( $<5$ days), persistent chest tube fluid output, time spent on the ventilator (positive pressure ventilation), and lung expansion and space management issues. These data
TABLE 2. Perioperative outcomes

\begin{tabular}{lc}
\hline \multicolumn{1}{c}{ Variable } & Patients $(\mathbf{n}=\mathbf{2 1 1})$ \\
\hline Operative time (min) & 60 \\
Vessels divided & \\
$\quad$ Arteries & 476 \\
$\quad$ Veins & 229 \\
Vessel size (mm) & \\
$\quad$ Mean & 6 \\
$\quad$ Range & $0.4-1.2$ \\
Vascular dehiscence & \\
$\quad$ Venous & 2 \\
$\quad$ Arterial & 0 \\
Estimated blood loss (mL) & 100 \\
Chest tube duration (d) & 5 \\
Length of stay (median) & 6 \\
Complications (\%) & 25.1 \\
Mortality (30-day) & $1(0.5 \%)$ \\
\hline
\end{tabular}

are comparable to the perioperative outcomes achieved with the use of endostaplers. ${ }^{9}$

\section{Morbidity and Mortality}

Two patients $(1.5 \%)$ in the VATS group required conversion to an open incision. No conversions were required secondary to intraoperative hemorrhage. Complications occurred in 53 patients $(25.1 \%)$ and are listed in Table 3. The most common complication was atrial fibrillation, which occurred in 14 patients $(6.6 \%)$. Prolonged air leaks longer than 5 days $(5.7 \%)$ and pneumonia $(5.2 \%)$ were the most common pulmonary complications. One patient $(0.5 \%)$ died perioperatively ( $\leq 30$ days) secondary to pneumonia with progression to acute respiratory distress syndrome. No postoperative complications occurred that were directly attributable to the use of the LigaSure device.

\section{TABLE 3. Complications}

\begin{tabular}{lc}
\hline Complication type & Patients (n= 211) \\
\hline Cardiac & $15(7.1 \%)$ \\
Atrial fibrillation & $14(6.6 \%)$ \\
Congestive heart failure & $1(0.5 \%)$ \\
Ventricular arrhythmia & $1(0.5 \%)$ \\
Pulmonary & $39(18.5 \%)$ \\
Air leak & $12(5.7 \%)$ \\
Pneumonia & $11(5.2 \%)$ \\
Bronchoscopy & $6(2.8 \%)$ \\
Pneumothorax & $4(1.9 \%)$ \\
Respiratory failure & $13(6.2 \%)$ \\
Empyema & $2(0.9 \%)$ \\
Infectious & $13(6.2 \%)$ \\
Pneumonia & $11(5.2 \%)$ \\
Empyema & $2(0.9 \%)$ \\
Septicemia & $2(0.9 \%)$ \\
Total patients (n) & $53(25.1 \%)$ \\
\hline
\end{tabular}



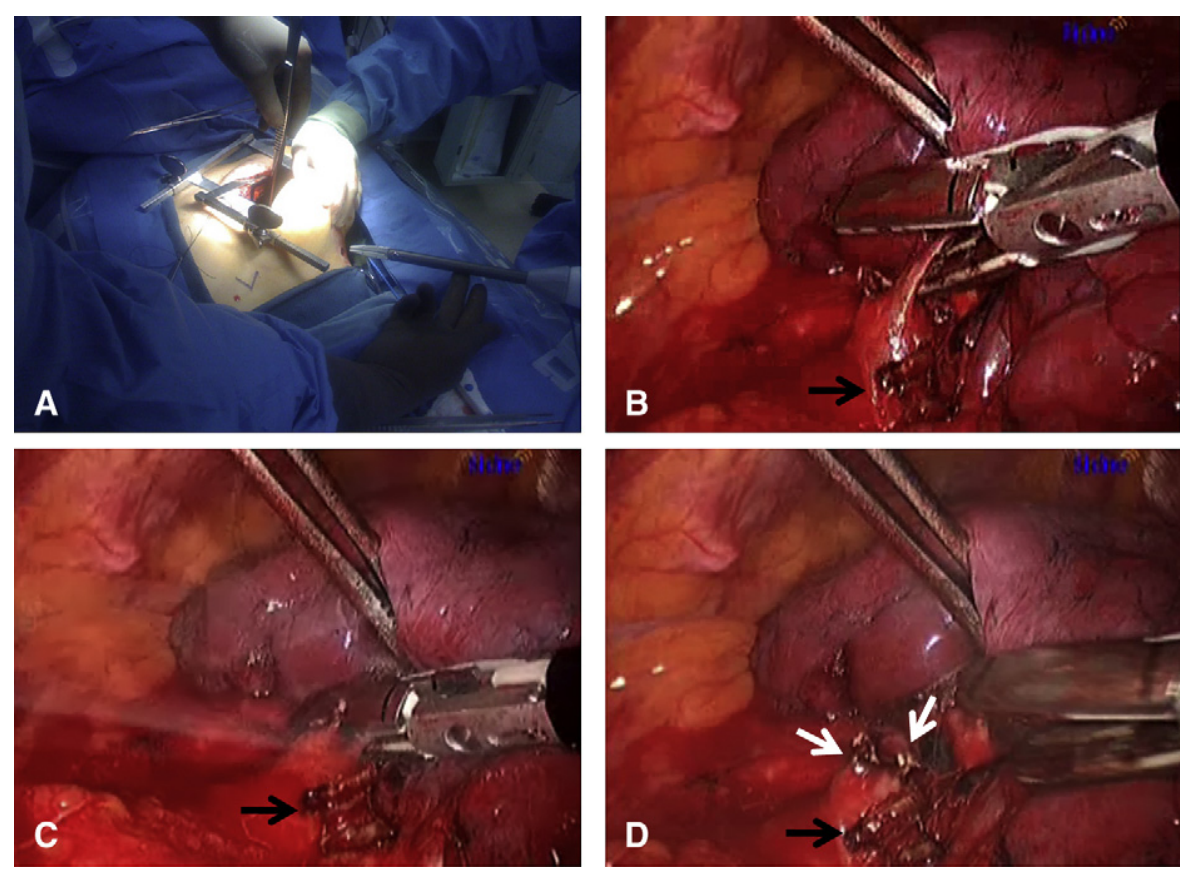

FIGURE 2. A, Introduction of LigaSure device by way of thoracoscopic port. B, Application of LigaSure device to anterior and apical arteries of left upper division. Note, divided superior pulmonary vein contribution to left upper division (black arrow). C, Following closure of jaws, vascular fusion is accomplished with 2 energy applications. D, Arterial (white arrows) and venous (black arrow) stumps after fusion and division.

\section{DISCUSSION}

With the introduction of VATS techniques, the endostapler has become the primary method of dividing both the pulmonary parenchyma and the vasculature. ${ }^{10,11}$ The use of energy-based devices for tissue fusion and division has gained increased popularity and use in a wide variety of intra-abdominal procedures. Few data currently exist regarding the use of this technology in thoracic applications, such as the division of the pulmonary vasculature during anatomic lung resection. Several energy-based fusion devices are currently available. Only the LigaSure device has been approved by the Food and Drug Administration for division of the intrathoracic vasculature and was, therefore, the device chosen for the present analysis.

The use of an energy-based device for vascular division within the thorax has been predicated on the findings of numerous animal studies, as well as a few clinical studies in humans. In preclinical swine models, the use of the bipolar electrocoagulation sealing devices has been shown to achieve a durable burst pressure strength of approximately $300 \mathrm{~mm} \mathrm{Hg}$, comparable to the burst pressures generated by vascular clips and staples, and far in excess of the pressures generated within the pulmonary vasculature. ${ }^{12}$ In a bovine study testing the burst pressures of $5-\mathrm{mm}$ arterial vessels treated with 4 different vascular sealing devices, the LigaSure device had greatest burst pressure $(385 \mathrm{~mm}$ $\mathrm{Hg})$ and shortest sealing time (10 seconds). ${ }^{13}$ Sugi and colleagues ${ }^{14}$ extended these studies to an evaluation of the fusion and division of the pulmonary vasculature in a live swine model and found that the LigaSure device safely divided pulmonary vessels $<5 \mathrm{~mm}$ in size, despite increasing pulmonary pressures to a challenge pressure of $75 \mathrm{~mm} \mathrm{Hg}$. Lacin and colleagues ${ }^{15}$ compared the vascular integrity of divided pulmonary arteries during lobectomy in sheep. They performed lobectomy in 12 sheep and compared the integrity of the fused vessels in those undergoing upper lobectomy $(n=6)$ and lower lobectomy $(n=6)$. Among the sheep undergoing lower lobectomy, the vascular dehiscence rate was $33 \%$ for the arteries and $50 \%$ for the veins larger than $9 \mathrm{~mm}$ in diameter. No vascular disruption was seen in the smaller arteries (mean, $5.7 \mathrm{~mm}$ ) and veins (mean, $4.6 \mathrm{~mm}$ ) encountered during upper lobectomy. These data suggested that the LigaSure device could be applied safely to smaller vessels up to $7 \mathrm{~mm}$ in size. ${ }^{15}$

In humans, the LigaSure device has demonstrated success in dividing major splenic, renal, and colonic arteries, and its use within the abdomen has been well-established. ${ }^{16-18}$ Several small series exploring its application within the chest have been published. The first case report of lung resection using the LigaSure was by Shigemura and colleauges. ${ }^{19}$ The same group, and others, have studied the use of bipolar tissue fusion during simple wedge resection, with excellent results. ${ }^{20-22}$ Albanese and colleagues ${ }^{23}$ were the first to report a series of anatomic lung resections performed in newborns for lesions diagnosed prenatally. In that series, lobar resection was performed in 14 infants for 
congenital cystic adenomatoid malformations or sequestration. No intraoperative or postoperative complications developed. Meehan and colleagues ${ }^{24}$ used both the LigaSure and the Gyrus PK device to perform robotic lung resections in 6 children without adverse events. Santini and colleagues $^{25}$ reported their experience in the use of the LigaSure device during lung resection in 36 patients, dividing vessels up to $7 \mathrm{~mm}$ in size. No major perioperative bleeding and no mortality occurred.

In the present series, the LigaSure vessel fusion technology was applied in 211 cases. Although 2 partial venous dehiscences were encountered using the LigaSure Impact handpiece, no vascular dehiscence were encountered using the LigaSure Atlas device during the division of 476 arteries and 229 veins ranging in size from $0.4 \mathrm{~mm}$ up to $1.2 \mathrm{~cm}$. It is believed that the uniform, wider jaw and greater sealing pressure of the LigaSure Atlas achieved a more reliable seal in this setting. This was particularly true for the thinwalled pulmonary veins. We also found that 2 applications of energy before division of the vessel achieved a more secure sealing zone. The partial vascular dehiscences encountered in the present series were readily controlled with suture ligation and were not associated with major blood loss, hemodynamic instability, or subsequent perioperative complications. These data suggest that the LigaSure Impact device can be safely and effectively used during vascular division for both anatomic segmentectomy and lobectomy.

The potential advantages of the bipolar tissue fusion technology include its ease of use in both open and thoracoscopic procedures. The smaller profile jaws are easier to pass around smaller vessels compared with the more bulky endostapling instruments. Excellent hemostasis can be achieved during the course of resection, with no bleeding typically seen from the margins of the resection. This device can also be used to develop the fissures and perform simple wedge resection, ${ }^{26}$ with minimal thermal spread to the tissues surrounding the sealing line $(2-3 \mathrm{~mm})$. Furthermore, a single hand-held device avoids the use of multiple reloadable stapler cartridges, thus reducing operative costs. The LigaSure device is priced at $\$ 350$. Assuming the use of 1.5 staplers per case ( $\$ 300$ each) and an average stapler cartridge cost of $\$ 100$, the total cost savings of performing the vascular divisions in 89 lobectomies (average 3.5 vessels/ case) and 122 segmentectomies (average 2.0 vessels per case) with the LigaSure would be in excess of $\$ 75,000$ for the patients treated in the present study. This did not account for the multiple staple loads used to develop the fissures that would also be amenable to division with the LigaSure, potentially increasing the cost savings to more $\$ 1000$ per patient. Such measures would further augment the reduced cost attributed to the VATS approach compared with standard thoracotomy. ${ }^{27,28}$

The present study was limited by its retrospective nature, introducing the possibility of bias in patient selection and perioperative management. No comparison groups were included to enhance the evaluation of the outcomes. The results of the present study should not be generalized to the use of any energy-based tissue fusion device during anatomic lung resection. Careful intraoperative decisionmaking and postoperative monitoring are required to ensure optimal patient outcomes.

\section{CONCLUSIONS}

The use of the LigaSure bipolar tissue fusion system represents a safe and effective technique for the division of both pulmonary arteries and veins (ideally $\leq 7 \mathrm{~mm}$ ) during anatomic lung resection. It can be considered a valid alternative to stapling technology in this setting. Although not specifically assessed in the present analysis, the use of this device could potentially lead to decreased operative costs by avoiding the use of the multiple endostapling cartridges typically required during anatomic lung resection. Larger prospective series with long-term results and cost/ benefit analyses are necessary to better define the utility of this technology during anatomic lung resection.

The authors wish to acknowledge the important contribution of Lorraine Ickes of the Thoracic Surgery Tumor Registry for her assistance in database management and analysis. We would also like to thank Alicia Oostdyk, Peg Reamer, Kimberly Burke, Lauren Matteo, Patricia Williams, and Maria Haffely for their assistance in organizing and analyzing hospital charts and providing timely updates regarding patient follow-up.

\section{References}

1. Szwerc MF, Landreneau RJ, Santos RS, Keenan RJ, Murray GF. Mini-thoracotomy combined with mechanically-stapled bronchial and vascular ligation for anatomical lung resection. Ann Thorac Surg. 2004;77:1904-9.

2. Wilkins EW Jr, Scannell JG, Craver JG. Four decades of experience with resections for bronchogenic carcinoma at the Massachusetts General Hospital. J Thorac Cardiovasc Surg. 1978;76:364-8.

3. Asamura H, Suzuki K, Kondo H, Tsuchiya R. Mechanical vascular division in lung resection. Eur J Cardiothorac Surg. 2002;21:879-82.

4. Weissberg D, Kaufman M. Suture closure versus stapling of bronchial stump in 304 lung cancer operations. Scand J Thorac Cardiovasc Surg. 1992;26: 125-7.

5. Ruffini E, Oliaro A, Manusco M, Cavallo A, Filosso P, Molinatti M, et al. Successful management of hemorrhage from the pulmonary artery stump after lobectomy for bronchogenic carcinoma. J Cardiovasc Surg. 1994;35:257-9.

6. Asamura H, Kondo H, Tsuchiya R. Management of the bronchial stump in pulmonary resections: A review of 533 consecutive recent bronchial closures. Eur J Cardiothorac Surg. 2000;17:106-10.

7. Casali G, Walker WS. Video-assisted thoracic surgery lobectomy: Can we afford it? Eur J Cardiothorac Surg. 2009;35:423-8.

8. Entezari K, Hoffmann P, Goris M, Peltier A, Van Velthoven R. A review of currently available vessel sealing systems. Minim Invasive Ther Allied Technol. 2007; 16:52-7.

9. Schuchert MJ, Pettiford BL, Pennathur A, Abbas G, Awais O, Close J, et al. Anatomic segmentectomy for stage I non-small cell lung cancer: Comparison of video-assisted thoracic surgery versus open approach. J Thorac Cardiovasc Surg. 2009;138:1318-25.

10. Allen MS, Deschamps C, Lee RE, Trastek VF, Daly RC, Pairolero PC. Video-assisted thoracoscopic stapled wedge excision for indeterminate pulmonary nodules. J Thorac Cardiovasc Surg. 1993;106:1048-52.

11. Lewis RJ, Caccavale RJ, Sisler GE, Mackenzie JW. Video-assisted thoracic surgical resection of malignant lung tumors. J Thorac Cardiovasc Surg. 1992;104:1679-85. 
12. Kennedy JS, Stranahan PL, Taylor KD, Chandler JG. High-burst-strength, feedback-controlled bipolar vessel sealing. Surg Endosc. 1998;12:876-8.

13. Lamberton GR, His RS, Jin DH, Lindler TU, Jellison FC, Baldwin DD. Prospective comparison of four laparoscopic vessel ligation devices. J Endourol. 2008; 22:2307-12.

14. Sugi K, Kaneda Y, Satoh Y, Murakami T. Effects of bipolar vessel sealing system in pulmonary resections. Jpn J Thorac Surg. 2003;56:551-4.

15. Lacin T, Batirel HF, Ozer K, Demirutku A, Ahiskali R, Yuksel M. Safety of a thermal vessel sealer on main pulmonary vessels. Eur J Cardiothorac Surg. 2007;31: 482-5.

16. Gelmini R, Romano F, Quaranta N, Caprotti R, Tazzioli G, Colombo G, et al. Sutureless and stapleless laparoscopic splenectomy using radiofrequency: LigaSure device. Surg Endosc. 2006;20:991-4.

17. Constant DL, Florman SS, Mendez F, Thomas R, Slakey DP. Use of the LigaSure vessel sealing device in laparoscopic liver-donor nephrectomy. Transplantation. 2004;78:1661-4.

18. Marcello PW, Roberts PL, Rusin LC, Holubkov R, Schoetz DJ. Vascular pedicle ligation techniques during laparoscopic colectomy: A prospective, randomized trial. Surg Endosc. 2006;20:263-9.

19. Shigemura N, Akashi A, Nakagiri T. New operative method for a giant bulla: Sutureless and stapleless thoracoscopic surgery using the LigaSure system. Eur J Cardiothorac Surg. 2002;22:646-8.

20. Shigemura N, Akashi A, Nakagiri T, Ohta M, Matsuda H. A new tissue-sealing technique using the LigaSure system for non-anatomical pulmonary resection: Preliminary results of sutureless and stapleless thoracoscopic surgery. Ann Thorac Surg. 2004;77:1415-9.

21. Sakuragi T, Okazaki Y, Mitsuoka M, Yamasaki F, Masuda M, Mori D, et al. The utility of a reusable bipolar sealing instrument, BiClamp ${ }^{\circledR}$, for pulmonary resection. Eur J Cardiothorac Surg. 2008;34:505-9.

22. Santini M, Fiorello A, Vicidomini G, Laperuta P. The use of LigaSure for preservation of a previous coronary artery bypass by using the left internal thoracic artery in a left upper lobectomy. J Thorac Cardiovasc Surg. 2008;136: 222-3.

23. Albanese CT, Sydorak RM, Tsao K, Lee H. Thoracoscopic lobectomy for prenatally diagnosed lung lesions. J Pediatr Surg. 2003;38:553-5.

24. Meehan JJ, Phearman L, Sandler A. Robotic pulmonary resections in children: Series report and introduction of a new robotic instrument. J Laparoendoscop Adv Surg Techniq. 2008;18:293-5.

25. Santini M, Vicidomini G, Baldi A, Gallo G, Laperuta P, Busiello L, et al. Use of an electrothermal bipolar sealing system in lung surgery. Eur J Cardiothorac Surg. 2006;29:226-30

26. Kovacs O, Szanto Z, Krasznai G, Herr G. Comparing bipolar electrothermal device and endostapler in endoscopic lung wedge resection. Interact Cardiovasc Thorac Surg. 2009;9:11-4.
27. Walker WS, Casali G. The VATS lobectomist: An analysis of costs and alterations in the traditional surgical working pattern in the modern surgical unit Thorac Surg Clin. 2008;18:281-7.

28. Park BJ, Flores RM. Cost-comparison of robotic video-assisted thoracic surgery and thoracotomy approaches to pulmonary lobectomy. Thorac Surg Clin. 2008; 18:297-300

\section{Discussion}

Dr Mark J. Krasna (Towson, Md). Very nice, Rod. How did you measure the vessel size when you talk about estimating the width, $6 \mathrm{~mm}$, and did you also use this technique to control the airways during your segmentectomies?

Dr Landreneau. First, that distance is $2 \mathrm{~cm}$ from that black mark distally to the black mark proximally up near the hinge point of the jaw. So, it was an estimate determined by the relationship of the vessel size to that $2 \mathrm{~cm}$ length on that 1020 device.

We did not use this on the airways.

Dr Todd L. Demmy (Buffalo, NY). I think this is great work, Rodney. We have been using the LigaSure as well for a number of years. You have to be careful if you are using the 5-mm device, because with less collagen in the vein wall, it can give away after division. Thus, I think this is great innovation using the larger device.

One other option I think the audience should know about is that the same company that makes the LigaSure makes a 3-row stapler. It is an older standard endovascular stapler, but it has a very small anvil, and you can pass that through the same type of incision, staple the proximal end with the 3-row stapler, and then you can use the LigaSure on the side that is coming out. That is another way to handle it. I wonder if you have ever tried that.

Dr Landreneau. I have not tried it because of just frustration with getting the staples involved with the LigaSure device. That is a problem if you use a combination stapler/LigaSure is that it will not complete the seal if there is even a small staple inside the jaw. We have tried to avoid that, but that is a reasonable method you have described if you can get enough distance between them. 